\title{
Gestión integral del riesgo y manejo de crisis en el contexto de la emergencia y desastres: una revisión bibliográfica
}

\author{
ADRIÁN TORRES ${ }^{(1)}$ y ALBERTO MATURANA ${ }^{(1)}$
}

\section{INTRODUCCIÓN}

Este estudio tiene como motivación central indagar en la literatura científica indexada, la importancia relativa asignada a las materias descritas en su título, a propósito del terremoto acaecido el 27 de febrero de 2010 en vastas zonas de Chile y los resultados preliminares de los seminarios realizados a este respecto por la Escuela de Salud Pública de la Universidad de Chile ${ }^{\mathrm{a}, \mathrm{b}}$, dirigidos y coordinados por el Dr. Alberto Maturana Palacios y su equipo ${ }^{c}$.

Las interrogantes que surgieron después de estos eventos son: ¿Existen estudios sobre la gestión del riesgo, el manejo de crisis, y la emergencia y desastres, en la literatura científica indexada? ¿Dan cuenta estos estudios de un determinado modelo, método o sistema, aplicable a las distintas situaciones de crisis?

Para responder estas preguntas se hizo una revisión de los trabajos existentes en la literatura científica indexada, ISI Web Of Knowledge, de todos los años disponibles y actualizados a mayo de 2010. Se utilizaron tres tópicos para la búsqueda: "gestión del riesgo", "gestión de crisis" y "emergencia y desastre". La búsqueda arrojó 20 artículos y sus resultados se presentan en dos tablas, la primera de las cuales da cuenta del nombre del estudio, el autor o los autores, la fuente, el número de pá- ginas, la fecha de la publicación y el ranking. En la segunda, por su parte, se da cuenta del numeral del estudio en un orden que va desde el más al menos actual, el nombre del trabajo, un pequeño resumen de contenido, el método utilizado y las palabras clave.

La importancia de la investigación y sus eventuales aportes son tratados en la parte discusión y son la respuesta a las preguntas planteadas.

\section{MATERIAL Y MÉTODO}

La revisión bibliográfica se hace a partir de las siguientes bases de dato: SCI-EXPANDED-1899-present; SSCI-1956-present; A\&HCI-1975-present; CPCI-S-1990-present; CPCI-SSH-1990-present; CCR-EXPANDED1986-present (includes Institute National de la Propriete Industrielle structure data back to 1840; e IC-1993-present.

Para la consulta se utilizaron los tópicos "gestión del riesgo", "gestión de crisis" y "emergencia y desastre". El criterio de selección fue que los artículos fueran publicaciones ISI.

De los artículos seleccionados se identificaron las categorías: Nombre del trabajo, autor o autores, fuente, páginas del artículo, fecha de publicación y ranking de impacto (Ver Ta-

(1) Escuela de Salud Pública. Facultad de Medicina. Universidad de Chile. Independencia 939. Santiago. Chile. adriantorr@gmail.com

(a) Seminario internacional, análisis y propuestas para una política nacional de gestión integral del riesgo y manejo de crisis, una visión académica, realizado los días 24 y 25 de marzo de 2010.

(b) II Seminario, análisis y propuestas para una política nacional de gestión integral del riesgo y manejo de crisis, hacia un país más seguro, realizado los días 18 y 19 de mayo de 2010.

(c) Dr. Alberto Maturana P., académico de la Escuela de Salud Pública y es Director de la Onemi. 
bla 1). Asimismo, se identificó el método y las palabras clave de cada artículo o estudio y se realizó un resumen de sus contenidos (Ver Tabla 2).

\section{RESULTADOS DE LA BÚSQUEDA}

Los resultados de la búsqueda en las categorías que se indican fueron los siguientes:

a) El ámbito de estudio tratado por los artículos, dentro de los temas consultados, es variado y cada uno representa uno diferente.

b) Las fuentes son variadas, no obstante to- das cumplen con los requisitos de rigurosidad científica propios de las publicaciones de la ISI WEB of Knowledge. c) El número de páginas promedio es de 10,6 siendo el mínimo de 3 y el máximo de 22, por lo que la desviación estándar es de 5,7. El número de páginas más recurrente (Moda) es de seis (6).

d) Las fechas de publicación van desde 1994 hasta 2010, siendo 2009, el año de publicación más recurrente.

e) Las categorías a las que pertenecen los estudios, categorizables, se sitúan mayoritariamente en los dos primeros cuartiles.

Tabla 1. De los estudios que abordan los tópicos: "gestión de riesgos", "manejo de crisis" y "emergencia y desastres", y sus principales datos de referencias .

\begin{tabular}{|c|c|c|c|c|c|c|}
\hline $\mathbf{N}^{\mathbf{o}}$ & Nombre de trabajo & Autor (es) & Fuente (source) & Páginas & $\begin{array}{c}\text { Fecha de } \\
\text { publicación }\end{array}$ & $\begin{array}{c}\text { Ranking por } \\
\text { Quartil[5] }\end{array}$ \\
\hline 1 & $\begin{array}{l}\text { Unmastered risks: } \\
\text { From crisis to } \\
\text { catastrophe An } \\
\text { economic and } \\
\text { management insight. }\end{array}$ & $\begin{array}{l}\text { Schenker-Wicki A, } \\
\text { Inauen M, } \\
\text { Olivares M. }\end{array}$ & $\begin{array}{l}\text { Journal Of Business } \\
\text { Research Volume: } \\
63 \text { Issue: } 4\end{array}$ & $337-346$ & 2010 & Business $=\mathrm{Q} 3$ \\
\hline 2 & $\begin{array}{l}\text { Crisis management } \\
\text { and safety } \\
\text { construction of } \\
\text { community. }\end{array}$ & Ma, Yingnan & $\begin{array}{l}\text { New Perspectives } \\
\text { On Risk Analysis } \\
\text { And Crisis } \\
\text { Response. Book } \\
\text { Series, Advances In } \\
\text { Intelligent Systems } \\
\text { Research. }\end{array}$ & $142-149$ & 2009 & $\mathrm{~S} / \mathrm{I}$ \\
\hline 3 & $\begin{array}{l}\text { Disaster } \\
\text { management: } \\
\text { findings from a } \\
\text { systematic review. }\end{array}$ & $\begin{array}{l}\text { Lettieri E, } \\
\text { Masella C, } \\
\text { Radaelli G }\end{array}$ & $\begin{array}{l}\text { Disaster prevention } \\
\text { and management } \\
\text { Volume: } 18 \text { Issue: } 2\end{array}$ & $117-136$ & 2009 & $\mathrm{~S} / \mathrm{I}$ \\
\hline 4 & $\begin{array}{l}\text { An inquiry into risk } \\
\text { prevention on ice } \\
\text { and snow disaster in } \\
\text { construction project. }\end{array}$ & Zhang XB & $\begin{array}{l}\text { Symposium } \\
\text { on Emergency } \\
\text { Management } 2009 \\
\text { (Isem'09) }\end{array}$ & $273-277$ & 2009 & S/I \\
\hline
\end{tabular}

d El orden de los artículos está dado, desde el más (2010) al menos reciente (1994). 


\begin{tabular}{|c|c|c|c|c|c|c|}
\hline $\mathbf{N}^{\mathbf{o}}$ & Nombre de trabajo & Autor (es) & Fuente (source) & Páginas & $\begin{array}{c}\text { Fecha de } \\
\text { publicación }\end{array}$ & $\begin{array}{c}\text { Ranking por } \\
\text { Quartil[5] }\end{array}$ \\
\hline 5 & $\begin{array}{l}\text { The feasibility study } \\
\text { of constructing } \\
\text { a long-term } \\
\text { mechanism of } \\
\text { catastrophic medical } \\
\text { assistance. }\end{array}$ & $\begin{array}{l}\text { Shen Shi-yong, } \\
\text { Zhang Jian-ming, } \\
\text { Zhang Sha }\end{array}$ & $\begin{array}{l}\text { Proceedings of } \\
2009 \text { International } \\
\text { Conference } \\
\text { on Public } \\
\text { Administration (5th), } \\
\text { Vol II }\end{array}$ & $462-467$ & 2009 & $\mathrm{~S} / \mathrm{I}$ \\
\hline 6 & $\begin{array}{l}\text { Advances in drama } \\
\text { theory for managing } \\
\text { global hazards } \\
\text { and disasters. } \\
\text { Part i: theoretical } \\
\text { foundation. }\end{array}$ & $\begin{array}{l}\text { Levy, Jason K., } \\
\text { Hipel, Keith W. }\end{array}$ & $\begin{array}{l}\text { Group Decision } \\
\text { and Negotiation } \\
\text { Volume: } 18 \text { Issue: } 4\end{array}$ & $303-316$ & 2009 & $\begin{array}{c}\text { Management } \\
=\mathrm{Q} 4 \text { y socials } \\
\text { sciences, } \\
\text { interdisciplinary } \\
\text { = Q3 }\end{array}$ \\
\hline 7 & $\begin{array}{l}\text { Emergency planning } \\
\text { and mitigation at } \\
\text { Vesuvius: A new } \\
\text { evidence-based } \\
\text { approach. }\end{array}$ & $\begin{array}{l}\text { Baxter PJ, } \\
\text { Aspinall WP, } \\
\text { Neri A, et al. }\end{array}$ & $\begin{array}{l}\text { Journal of } \\
\text { Volcanology } \\
\text { and Geothermal } \\
\text { Research Volume: } \\
178 \text { Issue: } 3 \\
\text { Special Issue: Sp. } \\
\text { Iss. Si }\end{array}$ & $454-473$ & 2008 & $\begin{array}{l}\text { Geosciences, } \\
\text { ultidisciplinary } \\
\text { = Q1 }\end{array}$ \\
\hline 8 & $\begin{array}{l}\text { Corporate crisis } \\
\text { management } \\
\text { Managing a major } \\
\text { crisis in a chemical } \\
\text { facility. }\end{array}$ & $\begin{array}{l}\text { Marwitz S, } \\
\text { Maxson N, } \\
\text { Koch B, et al. }\end{array}$ & $\begin{array}{l}\text { Journal of } \\
\text { Hazardous Materials } \\
\text { Volume: } 159 \text { Issue: } \\
1 \text { Special Issue: Sp. } \\
\text { Iss. Si }\end{array}$ & $92-104$ & 2008 & $\begin{array}{c}\text { Engineering, } \\
\text { civil = Q1; } \\
\text { engineering, } \\
\text { enviromental } \\
=\mathrm{Q} 1 ; \\
\text { enviromental } \\
\text { sciences = Q1 }\end{array}$ \\
\hline 9 & $\begin{array}{l}\text { Remote sensing } \\
\text { satellite imagery and } \\
\text { risk management: } \\
\text { image based } \\
\text { information } \\
\text { extraction. }\end{array}$ & $\begin{array}{l}\text { Bitelli G, } \\
\text { Gusella L }\end{array}$ & $\begin{array}{l}\text { Risk Analysis VI: } \\
\text { Simulation and } \\
\text { Hazard Mitigation }\end{array}$ & $149-158$ & 2008 & $\mathrm{~S} / \mathrm{I}$ \\
\hline 10 & $\begin{array}{l}\text { Role playing - } \\
\text { Applications in } \\
\text { hostage and crisis } \\
\text { negotiation skills } \\
\text { training. }\end{array}$ & $\begin{array}{l}\text { Van Hasselt VB, } \\
\text { Romano SJ, } \\
\text { Vecchi GM }\end{array}$ & $\begin{array}{l}\text { Behavior } \\
\text { Modification } \\
\text { Volume: } 32 \text { Issue: } 2\end{array}$ & $248-263$ & 2008 & $\begin{array}{l}\text { Psychology, } \\
\text { clinical = Q2 }\end{array}$ \\
\hline 11 & $\begin{array}{l}\text { Managing } \\
\text { construction } \\
\text { risks - planning, } \\
\text { prevention, and } \\
\text { control for an } \\
\text { effective risk- } \\
\text { management } \\
\text { program. }\end{array}$ & Galarnyk TG & $\begin{array}{l}\text { PCI Journal } \\
\text { Volume: } 52 \text { Issue: } 6\end{array}$ & $115-117$ & 2007 & $\begin{array}{c}\text { Construction } \\
\text { \& building } \\
\text { technology }= \\
\text { Q4 }\end{array}$ \\
\hline
\end{tabular}




\begin{tabular}{|c|c|c|c|c|c|c|}
\hline $\mathbf{N}^{\mathbf{o}}$ & Nombre de trabajo & Autor (es) & Fuente (source) & Páginas & $\begin{array}{c}\text { Fecha de } \\
\text { publicación }\end{array}$ & $\begin{array}{l}\text { Ranking por } \\
\text { Quartil[5] }\end{array}$ \\
\hline 12 & $\begin{array}{l}\text { Risk communication } \\
\text { for public health } \\
\text { emergencies. }\end{array}$ & Glik, Deborah C. & $\begin{array}{l}\text { Review of Public } \\
\text { Health Volume: } 28\end{array}$ & $33-54$ & 2007 & $\begin{array}{c}\text { Public, } \\
\text { enviromental } \\
\text { \& occupational } \\
\text { health = Q1 }\end{array}$ \\
\hline 13 & $\begin{array}{l}\text { Crisis intervention } \\
\text { and risk reduction. }\end{array}$ & Alexander D & $\begin{array}{l}\text { RISK21 - Coping } \\
\text { with Risks due to } \\
\text { Natural Hazards } \\
\text { in the } 21 \text { st } \\
\text { Century Book } \\
\text { Series: Proceedings } \\
\text { and Monographs in } \\
\text { Engineering, Water } \\
\text { and Earth Sciences }\end{array}$ & $51-56$ & 2006 & S/I \\
\hline 14 & $\begin{array}{l}\text { A propensity score } \\
\text { analysis of brief } \\
\text { worksite crisis } \\
\text { interventions after } \\
\text { the World Trade } \\
\text { Center disaster } \\
\text { implications for } \\
\text { intervention and } \\
\text { research. }\end{array}$ & $\begin{array}{l}\text { Boscarino JA, } \\
\text { Adams RE, } \\
\text { Foa EB, et al. }\end{array}$ & $\begin{array}{l}\text { Medical Care } \\
\text { Volume: } 44 \text { Issue: } 5\end{array}$ & $454-462$ & 2006 & $\begin{array}{c}\text { Health care } \\
\text { sciences \& } \\
\text { services = } \\
\text { Q1; public, } \\
\text { enviromental } \\
\text { \& occupational } \\
\text { health = Q1 }\end{array}$ \\
\hline 15 & $\begin{array}{l}\text { Rainfall estimate for } \\
\text { flood management } \\
\text { using meteorological } \\
\text { data from satellite } \\
\text { imagery. }\end{array}$ & $\begin{array}{l}\text { Budhakooncharoen } \\
\text { S }\end{array}$ & $\begin{array}{l}\text { Engineering, } \\
\text { Construction } \\
\text { and Operations } \\
\text { in Challenging } \\
\text { Environments: Earth } \\
\text { and Space 2004 }\end{array}$ & $215-222$ & 2004 & $\mathrm{~S} / \mathrm{I}$ \\
\hline 16 & $\begin{array}{l}\text { Flood risk } \\
\text { management with } \\
\text { photogrammetry and } \\
\text { space-borne remote } \\
\text { sensing in Hungary. }\end{array}$ & $\begin{array}{l}\text { Kugler Z, } \\
\text { Barsi A }\end{array}$ & $\begin{array}{l}\text { New Strategies for } \\
\text { European Remote } \\
\text { Sensing }\end{array}$ & $593-599$ & 2005 & $\mathrm{~S} / \mathrm{I}$ \\
\hline 17 & $\begin{array}{l}\text { Dam-break } \\
\text { flood emergency } \\
\text { management system. }\end{array}$ & $\begin{array}{l}\text { Rodrigues AS, } \\
\text { Santos MA, } \\
\text { Santos AD, et al. }\end{array}$ & $\begin{array}{l}\text { Water Resources } \\
\text { Management } \\
\text { Volume: } 16 \text { Issue: } 6\end{array}$ & $489-503$ & 2002 & $\begin{array}{c}\text { Engineering, } \\
\text { civil = Q1; } \\
\text { water resources } \\
=\mathrm{Q} 2\end{array}$ \\
\hline 18 & $\begin{array}{l}\text { Lessons of } \\
\text { emergency } \\
\text { management and its } \\
\text { philosophy in urban } \\
\text { earthquake disaster. }\end{array}$ & Kawata Y & $\begin{array}{l}\text { Structural Safety and } \\
\text { Reliability, Vols. 1-3 }\end{array}$ & $\begin{array}{l}1595- \\
1601\end{array}$ & 1998 & $\mathrm{~S} / \mathrm{I}$ \\
\hline
\end{tabular}




\begin{tabular}{|c|l|l|l|l|c|c|}
\hline $\mathbf{N}$ & Nombre de trabajo & Autor (es) & Fuente (source) & Páginas & $\begin{array}{c}\text { Fecha de } \\
\text { publicación }\end{array}$ & $\begin{array}{c}\text { Ranking por } \\
\text { Quartil[5] }\end{array}$ \\
\hline $\mathbf{1 9}$ & $\begin{array}{l}\text { Emergency } \\
\text { management for } \\
\text { coastal earthquake } \\
\text { disaster from the } \\
\text { viewpoint of the sea. }\end{array}$ & Tanaka I & $\begin{array}{l}\text { Proceedings of the } \\
\text { Seventh (1997) } \\
\text { International } \\
\text { Offshore and } \\
\text { Polar Engineering } \\
\text { Conference, Vol I, } \\
1997\end{array}$ & $29-34$ & 1997 & S/I \\
\hline $\mathbf{2 0}$ & $\begin{array}{l}\text { California american } \\
\text { trader oil-spill - } \\
\text { effective interagency } \\
\text { and public-private } \\
\text { collaboration in } \\
\text { environmental } \\
\text { disaster response. }\end{array}$ & $\begin{array}{l}\text { Gellert Ga, } \\
\text { Daugherty SJ, } \\
\text { Rabiee L, et al. }\end{array}$ & $\begin{array}{l}\text { Journal of } \\
\text { Environmental } \\
\text { Health Volume: } 57 \\
\text { Issue: 4 }\end{array}$ & $7-12$ & 1994 & $\begin{array}{c}\text { Q4; public, } \\
\text { enviromental } \\
\text { \& occupational } \\
\text { health=Q4 }\end{array}$ \\
\hline
\end{tabular}

Fuente: Elaboración propia

En cuanto a los contenidos de los estudios, los métodos utilizados y las palabras clave, el resultado fue el siguiente:

a) Los contenidos de los estudios son diversos $\mathrm{y}$, prácticamente, cada uno es un universo en sí mismo.

b) Los métodos utilizados son diversos, entre los que se encuentran: revisión bibliográfica, estudios y análisis descriptivos, análisis comparativo, análisis cualitativo y análisis cuantitativo, entre otros. Ver Tabla 2.

c) Los contenidos, dada su diversidad y extensión se exponen en la Tabla II.

d) Las palabras clave se extraen sólo de los once (11) estudios que las reportan y las de mayor recurrencia fueron, en orden de precedencia: management, disasters, risks, crisis, emergency, community $\mathrm{y}$ safety, entre otras.

Tabla 2. Resumen de contenidos, método y palabras claves asociadas a los estudios revisados ${ }^{\mathrm{e}-\mathrm{g}}$

\begin{tabular}{|c|c|c|c|c|}
\hline Doc. $\mathbf{N}^{o}$ & Cita del trabajo & Resumen de contenido & Método & Palabras clave \\
\hline 1 & $\begin{array}{l}\text { (Schenker-Wicki, } \\
\text { Inauen et al. 2010) }\end{array}$ & $\begin{array}{l}\text { El documento señala } \\
\text { que en contraste con la } \\
\text { sociología, la economía y, } \\
\text { sobre todo, la economía } \\
\text { de negocio aún no se ha } \\
\text { descubierto la gestión de } \\
\text { desastres como un tema } \\
\text { de investigación aun } \\
\text { cuando afecte muchas } \\
\text { áreas de la economía } \\
\text { dando indicios de su } \\
\text { carácter interdisciplinario. }\end{array}$ & Rational Choice & $\begin{array}{l}\text { Disaster management; } \\
\text { theoretical framework, } \\
\text { research, risk, } \\
\text { prevention, response. }\end{array}$ \\
\hline
\end{tabular}

(e) Cada artículo puede encontrarse en más de una publicación, por lo tanto tener más de un ranking.

(f) El resumen de contenido se hace, preferentemente, en base a los abstract de los artículos, por no disponer de las versiones completas de todos. La traducción es propia y para las palabras clave se mantuvo el idioma original.

(g) El orden de los artículos es el mismo usado en la tabla 1. 


\begin{tabular}{|c|c|c|c|c|}
\hline Doc. $\mathbf{N}^{\mathbf{0}}$ & Cita del trabajo & Resumen de contenido & Método & Palabras clave \\
\hline 2 & (Ma 2009) & $\begin{array}{l}\text { El documento se propone } \\
\text { establecer un nuevo } \\
\text { modelo de evaluación de } \\
\text { riesgos para evaluar la } \\
\text { gestión de emergencia de } \\
\text { la comunidad. }\end{array}$ & $\begin{array}{l}\text { Hierarchical Fuzzy } \\
\text { Analytical Network }\end{array}$ & $\begin{array}{l}\text { Community, crisis } \\
\text { management, safety } \\
\text { community construction, } \\
\text { crisis response reprint. }\end{array}$ \\
\hline 3 & $\begin{array}{l}\text { (Lettieri, Masella } \\
\text { et al. 2009) }\end{array}$ & $\begin{array}{l}\text { El paper trata de una } \\
\text { revisión sistemática de } \\
\text { la literatura sobre la } \\
\text { gestión de desastres en el } \\
\text { período 1980-2006. }\end{array}$ & $\begin{array}{l}\text { Análisis descriptivo como } \\
\text { un análisis temático para } \\
\text { proporcionar un estado } \\
\text { del arte de la literatura } \\
\text { internacional. }\end{array}$ & $\begin{array}{l}\text { Disasters; risk } \\
\text { management; defence } \\
\text { sector. }\end{array}$ \\
\hline 4 & (Zhang 2009) & $\begin{array}{l}\text { El paper da cuenta de } \\
\text { cómo reducir con eficacia } \\
\text { las consecuencias de los } \\
\text { desastres de hielo y nieve } \\
\text { sobre los proyecto de } \\
\text { construcción en China, } \\
\text { país que sufre muchos de } \\
\text { estos episodios. }\end{array}$ & $\begin{array}{l}\text { Se enfoca en un análisis } \\
\text { comprensivo acerca del } \\
\text { tema práctico de los } \\
\text { desastres de hielo y nieve. } \\
\text { Analiza los riesgos de los } \\
\text { proyectos de construcción y } \\
\text { realiza algunas propuestas. }\end{array}$ & \\
\hline 5 & $\begin{array}{l}\text { (Shen, Zhang et al. } \\
\text { 2009) }\end{array}$ & $\begin{array}{l}\text { La tesis después de un } \\
\text { análisis hace propuestas } \\
\text { para la construcción de } \\
\text { un sistema de asistencia } \\
\text { médica catastrófica } \\
\text { a largo plazo, como } \\
\text { la normalización de } \\
\text { gestión de crisis, el } \\
\text { establecimiento de un } \\
\text { fondo catastrófico médico } \\
\text { y el establecimiento de } \\
\text { personal médico, entre } \\
\text { otras. }\end{array}$ & $\begin{array}{l}\text { Análisis comparativo } \\
\text { sistemas de asistencia } \\
\text { médica catastrófica. } \\
\text { Analiza la evolución de } \\
\text { los derechos médicos de } \\
\text { seguros de personas en el } \\
\text { desastre catastrófico, que } \\
\text { es el soporte teórico del } \\
\text { mecanismo a largo plazo } \\
\text { de asistencia catastrófica } \\
\text { médica. }\end{array}$ & $\begin{array}{l}\text { Catastrophic medical } \\
\text { assistance, The } \\
\text { allocation of resources, } \\
\text { A long-term mechanis } \\
\text { catastrophic risk. }\end{array}$ \\
\hline 6 & $\begin{array}{l}\text { (Levy, Hipel et al. } \\
\text { 2009) }\end{array}$ & $\begin{array}{l}\text { El documento nos } \\
\text { propone utilizar, frente } \\
\text { al riesgo global y los } \\
\text { desafíos de la gestión de } \\
\text { desastres, la teoría del } \\
\text { drama como forma de } \\
\text { resolución de conflictos. }\end{array}$ & $\begin{array}{l}\text { Se basa en la teoría de } \\
\text { drama que constituye } \\
\text { un instrumento flexible } \\
\text { y poderoso para modelar } \\
\text { la decisión de grupos y } \\
\text { procesos de negociación que } \\
\text { implican peligros naturales, } \\
\text { artificiales y relacionados } \\
\text { con la salud, riesgo y } \\
\text { catástrofes en el entorno de } \\
\text { seguridad. }\end{array}$ & $\begin{array}{l}\text { Drama theory, } \\
\text { emergency, climate } \\
\text { change, disaster, conflict } \\
\text { resolution, decision } \\
\text { support. }\end{array}$ \\
\hline
\end{tabular}




\begin{tabular}{|c|c|c|c|c|}
\hline Doc. $\mathbf{N}^{0}$ & Cita del trabajo & Resumen de contenido & Método & Palabras clave \\
\hline 7 & $\begin{array}{l}\text { (Baxter, Aspinall et } \\
\text { al. 2008) }\end{array}$ & $\begin{array}{l}\text { El documento } \\
\text { nos propone usar, } \\
\text { frente a la necesidad } \\
\text { de desarrollar métodos } \\
\text { más robustos para la } \\
\text { evaluación de riesgo y } \\
\text { la toma de decisiones } \\
\text { en crisis volcánicas, el } \\
\text { nuevo paradigma: la } \\
\text { volcanología a base de } \\
\text { pruebas (evidence-based } \\
\text { volcanology). }\end{array}$ & $\begin{array}{l}\text { Análisis cuantitativo que } \\
\text { combina bancos de datos } \\
\text { sobre la vulnerabilidad del } \\
\text { entorno físico y humano } \\
\text { de desastres volcánicos } \\
\text { pasados con la investigación } \\
\text { teórica sobre el estado del } \\
\text { volcán, incluyendo pruebas } \\
\text { de campo sobre erupciones } \\
\text { anteriores así como el } \\
\text { modelismo de simulación } \\
\text { numérico de procesos } \\
\text { eruptivos. }\end{array}$ & $\begin{array}{l}\text { Planning; emergency, } \\
\text { volcano, eruption, } \\
\text { mitigation. }\end{array}$ \\
\hline 8 & $\begin{array}{l}\text { (Marwitz, Maxson } \\
\text { et al. 2008) }\end{array}$ & $\begin{array}{l}\text { El documento sostiene } \\
\text { que los sitios químicos } \\
\text { deberían tener planes } \\
\text { bien organizados } \\
\text { de respuesta a las } \\
\text { emergencias y para ello } \\
\text { propone un sistema de } \\
\text { manejo de incidentes a } \\
\text { nivel corporativo. }\end{array}$ & $\begin{array}{l}\text { Análisis descriptivo. } \\
\text { Describe un contorno para } \\
\text { la ayuda corporativa en caso } \\
\text { de un incidente mayor en un } \\
\text { sitio o durante el transporte. }\end{array}$ & \\
\hline 9 & $\begin{array}{l}\text { (Bitelli, Gusella } \\
\text { 2008) }\end{array}$ & $\begin{array}{l}\text { El documento plantea } \\
\text { que la introducción } \\
\text { de imágenes } \\
\text { multiespectrales de } \\
\text { satélite, de muy alta } \\
\text { resolución, ha llevado a } \\
\text { una nueva perspectiva } \\
\text { en los procesos de } \\
\text { valoración de riesgo, } \\
\text { mitigación y gestión. }\end{array}$ & $\begin{array}{l}\text { Se enfoca en realizar } \\
\text { un acercamiento para la } \\
\text { extracción de la información } \\
\text { y la clasificación } \\
\text { cuantitativa de la imagen y } \\
\text { otros datos de un desastre. }\end{array}$ & $\begin{array}{l}\text { Remote sensing, } \\
\text { disasters, damage } \\
\text { assessment, } \\
\text { segmentation. }\end{array}$ \\
\hline 10 & $\begin{array}{l}\text { (Van Hasselt, } \\
\text { Romano et al } \\
\text { 2008) }\end{array}$ & $\begin{array}{l}\text { El objetivo de este paper } \\
\text { es describir el desarrollo } \\
\text { y la validación de un } \\
\text { test de juego de roles, } \\
\text { expresamente adaptado } \\
\text { para negociadores de } \\
\text { aplicación de la ley. }\end{array}$ & $\begin{array}{l}\text { Juego de roles como } \\
\text { instrumento de negociación } \\
\text { de crisis extremistas. }\end{array}$ & $\begin{array}{l}\text { Role playing; hostage } \\
\text { negotiation; behavioral } \\
\text { assessment; law } \\
\text { enforcement training. }\end{array}$ \\
\hline 11 & (Galarnyk 2007) & $\begin{array}{l}\text { El artículo plantea } \\
\text { la diferencia de una } \\
\text { cultura empresarial que } \\
\text { maneja los riesgos de la } \\
\text { construcción, a través } \\
\text { de elementos de gestión } \\
\text { y sistemas de control } \\
\text { propios. }\end{array}$ & $\begin{array}{l}\text { Análisis en base a elementos } \\
\text { propios de la gestión y } \\
\text { sistemas de control. }\end{array}$ & $\begin{array}{l}\text { Accident; incident; } \\
\text { management; owners; } \\
\text { plants; risk; safety. }\end{array}$ \\
\hline
\end{tabular}




\begin{tabular}{|c|c|c|c|c|}
\hline Doc. $\mathbf{N}^{o}$ & Cita del trabajo & Resumen de contenido & Método & Palabras clave \\
\hline 12 & (Glik 2007) & $\begin{array}{l}\text { Este documento define } \\
\text { la comunicación de } \\
\text { riesgo de crisis y repasa } \\
\text { la literatura de cuatro } \\
\text { disciplinas diferentes } \\
\text { que informan sobre las } \\
\text { comunicaciones de riesgo } \\
\text { de crisis, especialmente } \\
\text { en la práctica de la salud } \\
\text { pública. }\end{array}$ & $\begin{array}{l}\text { Revisión bibliográfica. Se } \\
\text { repasa la literatura de cuatro } \\
\text { disciplinas diferentes que } \\
\text { informan comunicaciones } \\
\text { de riesgo de crisis. Estos } \\
\text { son la comunicación de } \\
\text { riesgo (a) ambiental, (b) } \\
\text { la gestión de desastres, (c) } \\
\text { la promoción de salud y la } \\
\text { comunicación y medios de } \\
\text { comunicación (d) y estudios } \\
\text { de comunicación. }\end{array}$ & $\begin{array}{l}\text { Health ommunication; } \\
\text { disasters; preparedness. }\end{array}$ \\
\hline 13 & (Alexander 2006) & $\begin{array}{l}\text { El documento aborda } \\
\text { el estado actual y las } \\
\text { futuras perspectivas } \\
\text { de la planificación de } \\
\text { emergencias. }\end{array}$ & $\begin{array}{l}\text { Revisión bibliográfica. } \\
\text { Se trata el estado actual } \\
\text { y las futuras perspectivas } \\
\text { de la planificación de } \\
\text { emergencias. }\end{array}$ & \\
\hline 14 & $\begin{array}{l}\text { (Boscarino, Adams } \\
\text { et al. 2006) }\end{array}$ & $\begin{array}{l}\text { Este estudio examina } \\
\text { el impacto de breves } \\
\text { intervenciones de } \\
\text { salud mental recibidas } \\
\text { por empleados en las } \\
\text { obras después del } \\
\text { desastre de World Trade } \\
\text { Center (WTCD) entre } \\
\text { trabajadores en Nueva } \\
\text { York. }\end{array}$ & $\begin{array}{l}\text { Encuesta a víctimas de } \\
\text { desastre }(9 / 11 \text { NYC) con el } \\
\text { fin de examinar el impacto } \\
\text { de breves intervenciones } \\
\text { de salud mental recibidas } \\
\text { por empleados en las obras } \\
\text { después del desastre. }\end{array}$ & $\begin{array}{l}\text { Emergency services, } \\
\text { crisis interventions, } \\
\text { community disasters, } \\
\text { mental health, alcohol } \\
\text { abuse, occupational } \\
\text { medicine, propensity } \\
\text { scores. }\end{array}$ \\
\hline 15 & $\begin{array}{l}\text { (Budhakooncharoen } \\
\text { 2004) }\end{array}$ & $\begin{array}{l}\text { Este artículo presenta } \\
\text { una investigación sobre } \\
\text { la estimación de lluvia } \\
\text { para la gestión de } \\
\text { inundaciones a partir de } \\
\text { datos meteorológicos } \\
\text { entregados por el } \\
\text { Satélite Geoestacionario } \\
\text { Meteorológico de Japón, } \\
\text { GMS- }\end{array}$ & $\begin{array}{l}\text { Relación entre la } \\
\text { precipitación moderada y } \\
\text { no moderada, a partir de } \\
\text { los datos entregados por } \\
\text { las estaciones sinópticas } \\
\text { y la imagen tomada por el } \\
\text { Satélite Geoestacionario } \\
\text { Meteorológico de Japón, } \\
\text { GMS-5. }\end{array}$ & \\
\hline 16 & $\begin{array}{l}\text { (Kugler, Barsi } \\
\text { 2005) }\end{array}$ & $\begin{array}{l}\text { El documento expone } \\
\text { un modelo húngaro de } \\
\text { análisis hidrológico que } \\
\text { investiga el riesgo de } \\
\text { inundación sobre la base } \\
\text { de mapas topográficos. }\end{array}$ & $\begin{array}{l}\text { Modelo de elevación digital } \\
\text { para simular acontecimiento } \\
\text { de inundación a través de } \\
\text { fotografía digital. Trazado } \\
\text { de mapa de riesgo. }\end{array}$ & \\
\hline
\end{tabular}




\begin{tabular}{|c|c|c|c|c|}
\hline Doc. $\mathbf{N}^{\mathbf{0}}$ & Cita del trabajo & Resumen de contenido & Método & Palabras clave \\
\hline 17 & $\begin{array}{l}\text { (Rodrigues, Santos } \\
\text { et al. 2002) }\end{array}$ & $\begin{array}{l}\text { En este artículo se } \\
\text { presenta un sistema } \\
\text { a base de Internet } \\
\text { que apoya la gestión } \\
\text { de emergencia de } \\
\text { inundación a las } \\
\text { autoridades portuguesas } \\
\text { de agua, propietarios } \\
\text { de presa y el sistema de } \\
\text { protección civil. }\end{array}$ & $\begin{array}{l}\text { La Planificación de } \\
\text { emergencia a través de un } \\
\text { sistema a base de Internet } \\
\text { que apoya la gestión de } \\
\text { emergencia de inundación. }\end{array}$ & \\
\hline 18 & (Kawata 1998) & $\begin{array}{l}\text { El documento presenta } \\
\text { el entorno de la gestión } \\
\text { de emergencia y sus } \\
\text { lecciones. Se define la } \\
\text { mitigación social como } \\
\text { la tecnología humana que } \\
\text { incluye infraestructura } \\
\text { ecológica. }\end{array}$ & $\begin{array}{l}\text { Revisión y discusión } \\
\text { bibliográfica. }\end{array}$ & \\
\hline 19 & (Tanaka 1997) & $\begin{array}{l}\text { Descripción de las } \\
\text { discusiones y ofertas de } \\
\text { los distintos estudios } \\
\text { surgidos después del } \\
\text { terremoto de Hyogo, } \\
\text { ocurrido en Japón el } 17 \\
\text { de enero de } 1995 \text { con una } \\
\text { magnitud } 7.2 \text { en la escala } \\
\text { de Richter. }\end{array}$ & Revisión bibliográfica & \\
\hline 20 & $\begin{array}{l}\text { (Gellert, Daugherty } \\
\text { et al. 1994) }\end{array}$ & $\begin{array}{l}\text { Este paper examina } \\
\text { la colaboración } \\
\text { de interagencia de } \\
\text { organizaciones públicas } \\
\text { y privadas durante el } \\
\text { derrame de petróleo } \\
\text { producido por el } \\
\text { petrolero mercante } \\
\text { americano en la Playa } \\
\text { Huntington, California, } \\
\text { en } 1990 \text {. }\end{array}$ & $\begin{array}{l}\text { Cualitativo: entrevistas } \\
\text { a informantes clave. } \\
\text { Cuantitativo: formulario de } \\
\text { evaluación de riesgos. }\end{array}$ & \\
\hline
\end{tabular}

Fuente: Elaboración propia

\section{DISCUSION}

La anterior revisión bibliográfica pone de manifiesto que el impacto producido por los desastres -tanto en el hombre como en su entorno por eventos de origen natural o antrópi- co- ha generado una reciente pero creciente preocupación en la literatura científica indexada, por los temas relacionados con la gestión del riesgo, el manejo de crisis y la emergencia y desastres, temas tratados en este artículo. El hecho que el más antiguo de los estudios co- 
rresponda a 1994 y que la mayor producción de este tipo de trabajo esté concentrada en el año 2009 , lo refleja en forma clara y consistente $^{\mathrm{h}}$. Esta nos muestra el interés existente entre los investigadores, sobre los tópicos tratados, aplicados a situaciones y ámbitos muy diversos, relacionados con desastres de origen natural, tales como: inundaciones, terremotos, erupción de volcanes, crecidas de ríos, aludes de nieve, etc., pero también por crisis generadas por el hombre, tales como el calentamiento global, el terrorismo o el derrame de petróleo, lo que implica una importante dispersión de temas. Cada artículo pone énfasis en un método, un modelo o un sistema de soporte para la toma de decisiones en situaciones extremas como las mencionadas, lo que también implica una gran dispersión de propuestas ${ }^{\mathrm{j}}$. Llama la atención la cantidad de disciplinas que quieren hacerse cargo de estos problemas para proponernos alguna solución, entre las que destacan: Las ciencias de la ingeniería, salud pública y ocupacional, ciencias del medioambiente, geociencias, psicología clínica, ciencias sociales interdisciplinaria y más recientemente las ciencias de la economía y la administración ${ }^{\mathrm{k}}$. Eso explica la diversidad de métodos usados a través de los cuales se arriba a las distintas conclusiones. Entre los métodos utilizados se encuentran la revisión bibliográfica sistemática, métodos cualitativos y cuantitativos, la Teoría del drama y Teoría del juego, sistemas de predicción meteorológica con soporte satelital, entre otros, cuestión que implica una gran gama de posibilidades, de acuerdo al problema que se quiere resolver ${ }^{1}$. Las categorías a las que pertenecen los estudios categorizables se sitúan mayoritariamente en los dos primeros cuartiles, lo que significa un reconocimiento expreso de la comunidad científica que está ocupada de estas materias, cuestión que los convierte en una fuente importante de desarrollo del conocimiento y de consulta obligada, para todos aquellos que quieren profundizar en estos distintos tópicos ${ }^{\mathrm{m}}$.

\section{REFERENCIAS $^{\mathrm{n}}$}

1. ALEXANDER, D., 2006. Crisis intervention and risk reduction.

2. BAXTER, P., ASPINALL, W., NERI, A., ZUCCARO, G., SPENCE, R. and CIONI, R., 2008. Emergency planning and mitigation at Vesuvius: A new evidence-based approach. Journal of Volcanology and Geothermal Research, 178(3), 454-473.

3. BITELLI, G. and GUSELLA, L., 2008. Remote sensing satellite imagery and risk management: image based information extraction. Southampton: Wit Press/Computational Mechanics Publications.

4. BOSCARINO, J., ADAMS, R., FOA, E. and LANDRIGAN, P., 2006. A propensity score analysis of brief worksite crisis interventions after the World Trade Center disaster implications for intervention and research. [Philadelphia]: Lippincott Williams Wilkins.

5. BUDHAKOONCHAROEN, S., 2004. Rainfall estimate for flood management using meteorological data from satellite imagery. New York: Amer Soc Civil Engineers.

6. GALARNYK, T., 2007. Managing construction risks - Planning, prevention, and control for an effective risk-management program. PCI Journal, 52(6), 115-117.

7. GELLERT, G., DAUGHERTY, S., RABIEE, L., MAZUR, M. and MERRYMAN, R., 1994. California American Trader Oil-Spill - Effective Interagency And Public-Private Collaboration In Environmental Disaster Response. Journal of environmental health, 57(4), 7-12.

8. GLIK, D., 2007. Risk communication for public health emergencies. Annual Review of Public Health, 28, 33-54.

9. KAWATA, Y., 1998. Lessons of emergency management and its philosophy in urban earthquake disaster. Leiden: A A Balkema Publishers.

\footnotetext{
(h) Vid. Tabla 1

(i) Vid. Tabla 2

(j) Idem

(k) Vid. Tabla 1

(l) Vid. Tabla 2

(m) Idem
}

(n) El orden de las referencias bibliográficas está de acuerdo a lo arrojado por el refworks, herramienta utilizada para dicho propósito 
10. KUGLER, Z. and BARSI, A., 2005. Flood risk management with photogrammetry and space-borne remote sensing in Hungary. Rotterdam: Millpress Science Publishers.

11. LETTIERI, E., MASELLA, C. and RADAELLI, G., 2009. Disaster management: findings from a systematic review. Disaster Prevention and Management, 18(2), 117-136.

12. LEVY, J., HIPEL, K. and HOWARD, N., 2009. Advances in Drama Theory for Managing Global Hazards and Disasters. Part I: Theoretical Foundation. Group Decision and Negotiation, 18(4), 303-316.

13. MA, Y., 2009. Crisis Management and Safety Construction of Community.

14. MARWITZ, S., MAXSON, N., KOCH, B., AUKERMAN, T., CASSIDY, J. and BELONGER, D., 2008. Corporate crisis management Managing a major crisis in a chemical facility. [Amsterdam]: Elsevier.

15. RODRIGUES, A., SANTOS, M., SANTOS, A. and ROCHA, F., 2002. Dam-break flood emergency management system. Water Resources Management, 16(6), 489-503.
16. SCHENKER-WICKI, A., INAUEN, M. and OLIVARES, M., 2010. Unmastered risks: From crisis to catastrophe An economic and management insight. Journal of business research, 63(4), 337-346.

17. SHEN, S., ZHANG, J. and ZHANG, S., 2009. The Feasibility Study of Constructing a Long-term Mechanism of Catastrophic Medical Assistance. CHENGDU: UNIV ELECTRONIC SCIENCE TECHNOLOGY CHINA PRESS.

18. TANAKA, I., 1997. Emergency management for coastal earthquake disaster from the viewpoint of the sea. Golden: International Society Offshore Polar Engineers.

19. VAN HASSELT, V., ROMANO, S. and VECCHI, G., 2008. Role playing - Applications in hostage and crisis negotiation skills training. Behavior modification, 32(2), 248-263.

20. ZHANG, X., 2009. An Inquiry into Risk Prevention on Ice and Snow Disaster in Construction Project. Irvin: Sci Res Publ, Inc-Srp.

Recepción: 5 de agosto de 2010 Aprobación: 7 de diciembre de 2010

Usted puede comentar éste y otros artículos publicados en la Revista Chilena de Salud Pública, enviando un correo electrónico a revistasp@med.uchile.cl 